\title{
A Cluster Model of Temperament as an Indicator of Antidepressant Response and Symptom Severity in Major Depression
}

\author{
Vesa Paavonen ${ }^{1,2} \bowtie$, Olli Kampman ${ }^{1,4}$, Ari Illi ${ }^{1,6}$, Merja Viikki ${ }^{1,5}$, Eija Setälä-Soikkeli ${ }^{3}$, and Esa Leinonen ${ }^{1,2}$ \\ ${ }^{1}$ University of Tampere School of Medicine, Tampere, Finland \\ ${ }^{2}$ Tampere University Hospital, Department of Psychiatry, Tampere, Finland \\ ${ }^{3}$ Kanta-Häme Central Hospital, Department of Psychiatry, Hämeenlinna, Finland \\ ${ }^{4}$ Seinäjoki Hospital District, Department of Psychiatry, Seinäjoki, Finland \\ ${ }^{5}$ Tampere Mental Health Centre, Tampere, Finland \\ ${ }^{6}$ Satakunta Hospital District, Department of Psychiatry, Harjavalta, Finland
}

Objective Not enough is known about which patients suffering from major depressive disorder benefit from antidepressant drug treatment. Individual temperament is relatively stable over a person's lifespan and is thought to be largely biologically predefined. We assessed how temperament profiles are related to depression and predict the efficacy of antidepressant treatment.

Methods We recruited one hundred Finnish outpatients (aged 19 to 72) suffering from major depressive disorder, of whom 86 completed the 6-week study. We assessed their temperament features with the Temperament and Character Inventory and used cluster analysis to determine the patient's temperament profile. We also categorized the patients according to the vegetative symptoms of major depressive disorder.

Results There was an association between skewed temperament profile and severity of major depressive disorder, but the temperament profiles alone did not predict antidepressant treatment response. Those with higher baseline vegetative symptoms score had modest treatment response. Our model with baseline Montgomery Åsberg Depression Rating Scale (MADRS) vegetative symptoms, age and temperament clusters as explanatory variables explained $20 \%$ of the variance in the endpoint MADRS scores.

Conclusion The temperament clusters were associated both with severity of depression and antidepressive treatment response of depression. The effect of the temperament profile alone was modest but, combined with vegetative symptoms of depression, their explanatory power was more marked suggesting that there could be an association of these two in the biological basis of MDD.

Psychiatry Investig 2014;11:18-23

Key Words Depressive disorder, Temperament, TCI, Antidepressive agents, Treatment response.

\section{INTRODUCTION}

According to the psychobiological model of temperament and character ${ }^{1}$ the human temperament can be divided into four different independent dimensions and character into three different dimensions. The temperamental features are: 1) the behavior in relation to new or pleasure-producing stimuli (novelty seeking, NS), 2) behavioral inhibition in relation to

Received: March 5, 2013 Revised: June 14, 2013

Accepted: July 3, 2013 Available online: January 21, 2014

$\triangle$ Correspondence: Vesa Paavonen, MD

University of Tampere School of Medicine, Arvo Building, FI-33014 Tampere, Finland

Tel: +358-407389051, Fax: +358-335516164, E-mail: vesa.paavonen@uta.fi

(c) This is an Open Access article distributed under the terms of the Creative Commons Attribution Non-Commercial License (http://creativecommons.org/licenses/by$\mathrm{nc} / 3.0$ ) which permits unrestricted non-commercial use, distribution, and reproduction in any medium, provided the original work is properly cited. issues which may lead to negative consequences (harm avoidance, HA), 3) continuing of behavior that has earlier been successful in the hope of reward (reward dependence, RD), and the tendency to maintain certain behavior despite frustration (persistence, P). ${ }^{1}$ These temperament dimensions are suggested to be connected with central neurotransmitter circuits in the central nervous system: dopamine (novelty seeking), serotonin (harm avoidance) and norepinephrine (reward dependence). ${ }^{2}$ The three dimensions of character mature in adulthood and influence personal and social effectiveness by insight learning about self-concepts. Self-concepts vary according to the extent to which a person identifies the self as 1) an autonomous individual (self-directedness, SD), 2) an integral part of humanity (cooperativeness, C), and 3) an integral part of the universe as a whole (self-transcendence, ST). ${ }^{1}$

The Temperament and Character Inventory (TCI) has been 
used in general population studies and in studies including patients with major depressive disorder (MDD) to assess how different temperament dimensions are associated with this disorder. ${ }^{1}$ HA has reportedly been higher in MDD patients than in general population ${ }^{3-9}$ and has been state dependent in MDD. ${ }^{5,6} \mathrm{HA}$ has also been associated with depressive symptoms in general population. ${ }^{10-12} \mathrm{HA}, \mathrm{RD}$ and NS have been found to have trait-like characteristics that are related to the familial occurrence of depression. ${ }^{4}$ High HA has been reported to predict poor treatment outcome. ${ }^{8,13-15}$

HA also seems to have a trait-like characteristic, reflecting genetic susceptibility in depression prone subjects. ${ }^{4,16}$ It seems that $\mathrm{HA}$ is both a state- and trait-dependent variable in MDD. ${ }^{17}$ However, one study using the Hospital Anxiety and Depression Scale (HAD), which excludes somatic symptoms, found no association between HA and MDD. ${ }^{18}$ This finding may suggest that HA is connected specifically to the somatic component of MDD. The contribution of other temperament dimensions in MDD is somewhat controversial or limited. Low $\mathrm{RD}$ may be associated with MDD and depressive symptoms in general population, ${ }^{4}$ but the results are unequivocal. ${ }^{10,19} \mathrm{NS}$ seems to be state dependent in MDD and altogether lower in MDD patients. ${ }^{4,11}$ However, high NS has been associated with history of suicide attempts in general population. ${ }^{20}$ In one study $\mathrm{P}$ was a state marker in depression. ${ }^{17}$

In a study by Grucza et al. ${ }^{20}$ different combinations of temperament dimensions were associated with different depressive symptoms. It has been proposed that the symptoms of MDD consist of clusters, which are linked to distinct genetic mechanisms which when combined in one individual, can lead to a diagnosable psychopathology. ${ }^{21}$ Suzuki et al..$^{22}$ proposed a three-factor model of the MADRS to differentiate the vegetative symptoms (somatic symptoms) observed in a patient group. This three-factor model has been used in some studies with MDD patient samples. ${ }^{23-25}$ It has been proposed that the vegetative symptoms are connected to enhanced expression of $5 \mathrm{HT} 2 \mathrm{~A}$ receptors. ${ }^{25} \mathrm{We}$ found no studies addressing the association of temperament and vegetative symptoms of MDD. The present study analyzes if temperament profiles in association with vegetative symptoms explain the antidepressant treatment response in MDD patients and if the severity of depression is associated with current temperament clusters.

\section{METHODS}

A hundred Finnish outpatients were recruited from secondary outpatient services, primary health care and by newspaper advertisements during the years 2002-2006 in the area of Tampere in southern Finland. The study was approved by the local Human Subjects Review Committee and subjects participated having given informed, voluntary, written consent. The recruitment resulted in 41 female and 59 male outpatients, aged 19-72 yrs (mean 40.7 years, $S D \pm 14.0$ ). Patients met the criteria for major depressive episode according to the Diagnostic and Statistical Manual of Mental Disorders, fourth edition (DSM-IV). All patients were diagnosed by a psychiatrist and the severity of their depression was evaluated with the Montgomery-Åsberg Depression Rating Scale (MADRS). Those patients who scored 20 or higher at baseline MADRS were included in the study. Patients with severe somatic illness or medication affecting their mood, other significant psychiatric disorders (bipolar illness, psychosis or severe personality disorders) or patients with alcohol or substance abuse were excluded from the study. Eighty-six patients completed the entire study according to the protocol and were included in the final analysis. ${ }^{26}$ Study data was collected on three occasions. At the first visit basic sociodemographic data was collected: gender, age, marital status, education, workplace before the sick leave, somatic illnesses and their medications, other psychiatric disorders, and possible use of psychotrophic medications. The baseline MADRS form was scored and the patients completed the temperament section of the TCI questionnaire to assess the temperament profile. ${ }^{1}$ All patients were prescribed either citalopram, fluoxetine or paroxetine. Anxiolytics and sedative hypnotics as adjuvant treatment and other medication for concomitant general medical conditions were allowed. At the second visit, three weeks after initiation of treatment, patients' adherence to treatment and the dosage of the medications were checked. Compliance was evaluated by a medication diary kept by the patient. Treatment compliance was deemed sufficient if the patient had taken the medication on at least $80 \%$ of the days in the study period. At the third visit, six weeks after the initiation, patients' adherence was monitored again and the MADRS and TCI forms were completed again (endpoint data). In the case of possible dropouts the necessary patient information on the reasons for dropout was also collected. The temperament profiles were determined from the baseline TCI data. The vegetative symptoms were assessed as the sum of questions three to five in the MADRS. ${ }^{22}$ These are impaired sleep, impaired appetite, and inner tension.

\section{Statistical methods}

A two-step cluster analysis was used for the definition of the patient's temperament profile. In our cluster model, we decided to use three temperament dimensions, NS, HA and RD with their baseline scores. In the statistical analysis the patients were divided into three clusters.

The differences in continuous variables (MADRS total score, 
MADRS factor scores and age) between the clusters were calculated with ANOVA. The difference in MADRS change between low and high vegetative symptom groups was analyzed with t-test. Differences between grouping variables were calculated with $\chi^{2}$-statistics. Non-parametric tests were used in comparisons in ordinal variables between different clusters (used medications, patient compliance). Pearson's correlation coefficients were calculated between MADRS total score and vegetative symptom score, both at baseline and endpoint.

In the multivariate analysis all variables included in the study and likely to have an impact on either depression severity or treatment response were used in the models. The effect of background variables on clusters was analyzed with a multinominal logistic regression model. Gender, age, severity of depression, antidepressant taken and dose, subjective adherence to treatment, and earlier depression episode were used as explanatory variables. A linear regression model (ANCOVA) was used for testing the effects of temperament clusters and other variables on MADRS endpoint scores. The first model included temperament clusters and age, and the second model temperament clusters, age and the MADRS vegetative symptoms (questions 3-5) at baseline as explanatory variables. All analyses were performed with SPSS for Windows software (version 17.0).

\section{RESULTS}

The cluster analysis resulted in the following clusters: LNS/ HHA/LRD, INS/HHA/HRD and HNS/LHA/(HRD) with $\mathrm{H}$ indicating high level, L low level and I intermediate level on the temperament dimensions. In the third cluster RD did not reach statistical significance in the clustering model. In the first cluster we discovered the most robust slope in the NS and HA at baseline. In the second cluster there were elevated points in HA. The results of the cluster analysis and MADRS scores in each cluster are presented in Table 1.

There were no differences in the distributions of gender between the clusters ( $p=0.23$, chi-square test). The patients in cluster 1 were older than in other clusters (age mean $\pm S D$, cluster $1=45.9 \pm 11.4$, cluster $2=37.4 \pm 14.4$, cluster $3=38.2 \pm 15.3$; $\mathrm{p}=0.03$, ANOVA). There was no difference between the clusters in the dosages of the medications taken in weeks one to three ( $\mathrm{p}=0.48$, Kruskal Wallis test), nor in compliance to treatment ( $p=0.69)$. Gender, severity of depression, antidepressant taken and dose, adherence to treatment, and earlier depression episode had no effect on the clusters in the multinominal regression model. Age of the patients had a marginal effect on clusters $(\mathrm{p}=0.051)$ in the multinominal regression model.

The correlations between MADRS vegetative symptom score with MADRS total score were at baseline 0.73 , $(\mathrm{p}<0.001)$ and at endpoint $0.76,(\mathrm{p}<0.001)$. The MADRS vegetative symptom score at baseline had a moderate correlation with MADRS endpoint scores ( $\mathrm{r}=0.38, \mathrm{p}<0.001)$, and a non-significant correlation with MADRS score change $(r=0.13, \mathrm{p}=0.26)$. We also analyzed the MADRS score change between patients with low $(1-7, n=50)$ and high ( 8 or more, $n=48)$ vegetative symptoms. The difference was close to significant [MADRS

Table 1. Results of the cluster analysis. All scores except response percentages are indicated as mean $\pm S D$

\begin{tabular}{|c|c|c|c|}
\hline Cluster & LNS/HHA/LRD, N=33 & INS/HHA/HRD, N=35 & $\mathrm{HNS} / \mathrm{LHA} /(\mathrm{HRD}), \mathrm{N}=30$ \\
\hline \multicolumn{4}{|l|}{ TCI baseline score } \\
\hline NS & $13.9 \pm 5.2$ & $19.6 \pm 7.1$ & $25.7 \pm 4.9$ \\
\hline $\mathrm{HA}$ & $26.8 \pm 6.1$ & $27.2 \pm 3.6$ & $16.0 \pm 4.2$ \\
\hline $\mathrm{RD}$ & $11.8 \pm 2.2$ & $18.2 \pm 2.5$ & $16.6 \pm 3.6$ \\
\hline Baseline MADRS* & $28.3 \pm 6.1$ & $27.3 \pm 5.7$ & $25.0 \pm 4.4$ \\
\hline Factor 1 (dysphoria) & $8.5 \pm 2.6$ & $7.8 \pm 2.2$ & $7.4 \pm 1.4$ \\
\hline Factor 2 (retardation) & $11.7 \pm 2.2$ & $12.1 \pm 2.7$ & $11.0 \pm 2.9$ \\
\hline Factor 3 (vegetative symptoms) & $7.8 \pm 3.1$ & $7.4 \pm 2.8$ & $6.4 \pm 2.7$ \\
\hline Endpoint MADRS** & $14.1 \pm 9.1$ & $13.5 \pm 8.3$ & $8.3 \pm 5.5$ \\
\hline Factor 1 (dysphoria) & $3.7 \pm 3.2$ & $3.9 \pm 2.7$ & $2.5 \pm 1.9$ \\
\hline Factor 2 (retardation) ${ }^{* * * * *}$ & $6.3 \pm 4.5$ & $6.0 \pm 3.7$ & $3.4 \pm 3.0$ \\
\hline Factor 3 (vegetative symptoms) & $3.5 \pm 2.3$ & $3.7 \pm 2.9$ & $2.4 \pm 1.9$ \\
\hline MADRS score change*** & $14.2 \pm 7.4$ & $14.3 \pm 8.0$ & $16.7 \pm 6.0$ \\
\hline Response (percentage decline in MADRS) ${ }^{* * * *}$ & $51.6 \%$ & $51.9 \%$ & $66.7 \%$ \\
\hline
\end{tabular}

${ }^{*} \mathrm{p}=0.05$ between groups (ANOVA), ${ }^{* *} \mathrm{p}=0.01$ between groups (ANOVA), ${ }^{* * *} \mathrm{p}=0.36$ between groups (ANOVA), ${ }^{* * * *} \mathrm{p}=0.04$ between groups (ANOVA), ${ }^{* * * * *} \mathrm{p}=0.01$ between groups (ANOVA). TCI: Temperament and Character Inventory, MADRS: Montgomery-Åsberg Depression Rating Scale, NS: novelty seeking, HA: harm avoidance, RD: reward dependence, with H indicating high level, L low level and I intermediate level on the temperament dimensions 
change, mean $( \pm S D)$, low symptoms $=13.5( \pm 5.5)$, high symptoms $=16.6( \pm 8.5), \mathrm{p}=0.052$, $\mathrm{t}$-test $]$. There were no differences in the MADRS vegetative symptom score at baseline or at endpoint between the different clusters $(\mathrm{p}=0.17$, baseline; $\mathrm{p}=0.14$, endpoint, ANOVA). There was a non-significant correlation between baseline MADRS dysphoria symptoms and MADRS score change ( $r=0.17, \mathrm{p}=0.11)$. MADRS endpoint scores were used as the outcome variable in two linear regression models. In the first model age and temperament clusters were used as explaining variables. This model explained $10 \%$ of the variance in the MADRS endpoint scores ( $p=0.04$; power 0.69 ). The clusters explained $9 \%(\mathrm{p}=0.02)$, and age explained $1 \%$ $(\mathrm{p}=0.36)$. In the second model, baseline MADRS vegetative symptoms, age and temperament clusters were used as explanatory variables. This model explained $20 \%$ of the variance in the MADRS endpoint scores $(\mathrm{p}=0.001$; power 0.96 for the complete model). In this model the baseline vegetative symptoms explained $12 \%(\mathrm{p}=0.001)$, age $0.2 \%(\mathrm{p}=0.70)$ and temperament clusters $5 \%(\mathrm{p}=0.12)$. Using the delta scores of MADRS as an outcome variable, and age and temperament clusters as explanatory variables (first model), and vegetative symptoms, age and temperament clusters as explanatory variables (second model) resulted in non-significant models (first model: $\eta \mathrm{p}^{2}=0.085, \mathrm{p}=0.061$, power $=0.61$; second model: $\eta \mathrm{p}^{2}=$ $0.096, \mathrm{p}=0.087$, power $=0.60$ ).

\section{DISCUSSION}

Our main hypothesis was that temperament clusters in patients with MDD explain the treatment response. In practice this means that different temperament profiles could function as a classifying factor and that MDD patients could be divided into different groups with different outcomes for antidepressant treatment. In our study we used primarily the MADRS endpoint scores as an outcome variable in the multivariate analyses. Using the delta scores of MADRS as an outcome variable resulted in non-significant models although there was a trend towards a better response in patients with high vegetative symptoms. The present results suggest that the combined effect of vegetative symptoms and temperament clusters is important in relation to the depression treatment outcome when measured as post-treatment symptoms. However, these factors showed a non-significant effect when predicting the change in depression scores during treatment. This finding may be due to both the temperament clusters and pretreatment vegetative symptoms representing depressive traits less connected with the magnitude of symptom alleviation during treatment.

The approach of using TCI temperament clusters for predicting the response to antidepressant treatment in MDD pa- tients is novel. In several studies individual dimensions of temperament have been used as precursors. Two earlier studies with general population samples have used combinations of high or low temperament traits for predicting different clinical features, but in these studies no cluster analysis method was used in classifying the temperament traits. ${ }^{18,20}$ This study did not include the character dimensions of the TCI (SD, C, ST) in the explanatory model. Adding the character traits to the predictors in the statistical model might have increased its predictive value regarding antidepressant response, since many studies have demonstrated that SD exhibits a state/trait marker in depression. ${ }^{9,27,28}$

There were some limitations concerning our patient sample and study setting. In contrast to some earlier studies, our patient sample comprised solely outpatients. This may have resulted in lower intensity of symptoms as reflected by the MADRS scores. Temperament profiles could have had more explanatory power if the patient sample had included inpatients with more severe depression. The patients were deemed compliant with medication if they took the prescribed medication at least $80 \%$ of the time, which can be regarded as a moderate level of treatment compliance, and the data were collected from patient reports, which in some cases may produce unreliable results. Nor did the patients receive any specific psychological treatment during the study, but were treated in a standard secondary outpatient setting.

As the relationship between temperament and vegetative symptoms of depression has not previously been studied, a post-hoc analysis with vegetative symptoms was performed in this study. This was done by separating the vegetative symptoms from the other symptoms of depression (dysphoria and retardation) which was based on the study by Suzuki et al. ${ }^{22}$ It has been proposed that the vegetative symptoms are connected to enhanced expression of 5-HT2A receptors. ${ }^{25}$

To assess patients' temperament profiles we used Cloninger's TCI, which has been widely used, validated and shown to be reliable in studies on general population and MDD patients. ${ }^{1}$ Temperament profiles were determined by clustering the distributions in the three temperament dimensions. Due to the limited sample size, the number of clusters was determined as three in the analysis to yield groups of reasonable size. The clustering method was able to differentiate between the three combinations of temperament traits, although in the third cluster the difference on the dimension $\mathrm{RD}$ did not reach statistical significance. It has been suggested that high RD correlates negatively with depressive symptoms, but the evidence is contradictory. ${ }^{3,4,9,10}$ The clusters differed on the dimension NS as it was low in cluster one, intermediate in cluster two and high in cluster three. The third cluster (HNS/LHA/HRD) probably reflects more impulsive depression, and diverges substan- 
tially from the typical temperament profile of an MDD patient, and in this study from the other two temperament clusters. This may explain why this subgroup of patients recovered better from the retardation symptoms than did the other patients. In addition, this group showed a higher percentage of MADRS changes. This difference in response could be due to the predictive effect of HA on MDD remission shown in our earlier report. ${ }^{29}$ In the first cluster we discovered the most distinct sloping in the distribution of the NS and HA dimensions at baseline. According to earlier reports the subgroup in this cluster has an increased risk for MDD and their depression is more disease-like. ${ }^{4-6,9}$ NS has been negatively associated with depressive mood state and tends to be at a low level in MDD patients. ${ }^{4}$ The clusters might thus reflect an underlying factor explaining clinically different symptoms profiles and course of depression.

In our study the temperament clusters were associated with both baseline and endpoint depressive symptoms and with the treatment response. The findings suggested that depression was most severe and difficult to treat in cluster one patients. In clusters one and two, in which HA scores were high, the response in percentage decline of MADRS scores was lower than in cluster three. These findings concur with those of earlier studies on the association between HA and depression. ${ }^{4,5,7-9,15,30}$

Although the depression vegetative symptom score is only a subscale of MADRS, it may be considered a separate dimension in depression symptomatology. ${ }^{22}$ MADRS total scores and vegetative symptoms showed a strong correlation at both baseline and endpoint. However, the correlation between MADRS endpoint total score and baseline vegetative symptom score was much lower, suggesting that the vegetative symptoms are a separate entity within depressive symptomatology.2. Therefore we considered it justified to study the impact of baseline vegetative symptoms on total symptoms at endpoint.

In the linear regression models our aim was to predict the treatment response in MDD patients. The first model was designed to reveal the impact of temperament clusters on treatment outcome. When patient's age was also taken into account as an explanatory variable, the temperament clusters had only modest explanatory power. Age as such did not function as an explaining variable in this model at a significant level. In the second model we wanted to ascertain if there was an interaction with temperament clusters and vegetative symptoms of MDD. Therefore we added the vegetative symptom scores to the model as an explanatory variable. In this model, the vegetative symptoms explained about twice as much as the clusters of the variance of endpoint MADRS scores. However, the whole model explained as much as one fifth of the variance in response to SSRI treatment. The role of the interaction between temperament clusters and vegetative symptoms on treatment result has to be interpreted cautiously, as the impact of clusters on treatment response in the final model was marginal. It seems that the vegetative symptoms of depression, in addition to a certain temperament profile, is a marked predictor for antidepressive treatment outcome. It is, however, possible that the vegetative symptoms alone have a more marked impact in both severity and response of depression compared to temperament. Even though the differences between the clusters in depression severity were marginal, our findings suggest an association between skewed temperament profile and severity of MDD. It is possible that the temperament profile can function as a predisposing factor to depression or have an impact on the clinical profile and course of depression.

In conclusion our study showed that MDD patients could be divided into different temperament clusters with different severity and outcomes of antidepressant treatment. The vegetative symptoms of depression combined with temperament profiles and age predicted antidepressant treatment response. The effect of the temperament profile alone was modest but, combined with vegetative symptoms of depression their explanatory power was more marked, suggesting that there could be an association between these two in the biological basis of MDD.

\section{Acknowledgments}

This study was supported by research grants by the Lilly Foundation Finland, and by medical funds of Kanta-Häme Central Hospital and Tampere University Hospital Districts.

\section{REFERENCES}

1. Cloninger CR, Svrakic DM, Przybeck TR. A psychobiological model of temperament and character. Arch Gen Psychiatry 1993;50:975-990.

2. Cloninger CR. A unified biosocial theory of personality and its role in the development of anxiety states. Psychiatr Dev 1986;4:167-226.

3. de Winter RFP, Wolterbeek R, Spinhoven P, Zitman FG, Goekoop JG. Character and temperament in major depressive disorder and a highly anxious-retarded subtype derived from melancholia. Compr Psychiatry 2007;48:426-435.

4. Farmer A, Mahmood A, Redman K, Harris T, Sadler S, McGuffin P. A sib-pair study of the Temperament and Character Inventory scales in major depression. Arch Gen Psychiatry 2003;60:490-496.

5. Hansenne M, Reggers J, Pinto E, Kjiri K, Ajamier A, Ansseau M. Temperament and Character Inventory (TCI) and depression. J Psychiatr Res 1999;33:31-36.

6. Hirano S, Sato T, Narita T, Kusunoki K, Ozaki N, Kimura S, et al. Evaluating the state dependency of the Temperament and Character Inventory dimensions in patients with major depression: a methodological contribution. J Affect Disord 2002;69:31-38.

7. Kimura S, Sato T, Takahashi T, Narita T, Hirano S, Goto M. Typus melancholicus and the Temperament and Character Inventory personality dimensions in patients with major depression. Psychiatry Clin Neurosci 2000;54:181-189.

8. Marijnissen G, Tuinier S, Sijben AE, Verhoeven WM. The temperament and character inventory in major depression. J Affect Disord 
2002;70:219-223.

9. Richter J, Polak T, Eisenmann M. Depressive mood and personality in terms of temperament and character among the normal population and depressive inpatients. Pers Indiv Differ 2003;35:917-927.

10. Naito M, Kijima N, Kitamura T. Temperament and Character Inventory (TCI) as predictors of depression among Japanese college students. J Clin Psychol 2000;56:1579-1585.

11. Jurado D, Gurpegui M, Moreno O, Fernandez MC, Luna JD, Galvez R. Association of personality and work conditions with depressive symptoms. Eur Psychiatry 2005;20:213-222.

12. Jylha $P$, Isometsa E. Temperament, character and symptoms of anxiety and depression in the general population. Eur Psychiatry 2006;21:389395.

13. Abrams KY, Yune SK, Kim SJ, Jeon HJ, Han SJ, Hwang J, et al. Trait and state aspects of harm avoidance and its implication for treatment in major depressive disorder, dysthymic disorder, and depressive personality disorder. Psychiatry Clin Neurosci 2004;58:240-248.

14. Sato T, Hirano S, Narita T, Kusunoki K, Kato J, Goto M, et al. Temperament and character inventory dimensions as a predictor of response to antidepressant treatment in major depression. J Affect Disord 1999;56: 153-161.

15. Kampman O, Poutanen O. Can onset and recovery in depression be predicted by temperament? A systematic review and meta-analysis. J Affect Disord 2011;135:20-27.

16. Sato T, Narita T, Hirano S, Kusunoki K, Goto M, Sakado K, et al. Factor validity of the temperament and character inventory in patients with major depression. Compr Psychiatry 2001;42:337-341.

17. Hansenne M, Bianchi J. Emotional intelligence and personality in major depression: trait versus state effects. Psychiatry Res 2009;166:63-68.

18. Matsudaira T, Kitamura T. Personality traits as risk factors of depression and anxiety among Japanese students. J Clin Psychol 2006;62:97109.

19. Peirson AR, Heuchert JW. The relationship between personality and mood: Comparison of the BDI and the TCI. Pers Indiv Differ 2001;30: 391-399.

20. Grucza RA, Przybeck TR, Spitznagel EL, Cloninger CR. Personality and depressive symptoms: a multi-dimensional analysis. J Affect Disord 2003;74:123-130.

21. Ginsburg BE, Werick TM, Escobar JI, Kugelmass S, Treanor JJ, Wendtland L. Molecular genetics of psychopathologies: a search for simple answers to complex problems. Behav Genet 1996;26:325-333.

22. Suzuki A, Aoshima T, Fukasawa T, Yoshida K, Higuchi H, Shimizu T, et al. A three-factor model of the MADRS in major depressive disorder. Depress Anxiety 2005;21:95-97.

23. Higuchi H, Sato K, Yoshida K, Takahashi H, Kamata M, Otani K, et al. Predictors of antidepressant response to fluvoxamine obtained using the three-factor structures of the Montgomery and Asberg Depression Rating Scale for major depressive disorders in Japanese patients. Psychiatry Clin Neurosci 2008;62:301-306.

24. Higuchi H, Sato K, Yoshida K, Takahashi H, Kamata M, Otani K, et al. No predictors of antidepressant patient response to milnacipran were obtained using the three-factor structures of the Montgomery and Asberg Depression Rating Scale in Japanese patients with major depressive disorders. Psychiatry Clin Neurosci 2008;62:197-202.

25. Kamata M, Suzuki A, Yoshida K, Takahashi H, Higuchi H, Otani K. Genetic polymorphisms in the serotonergic system and symptom clusters of major depressive disorder. J Affect Disord 2011;135:374-376.

26. Illi A, Setala-Soikkeli E, Viikki M, Poutanen O, Huhtala H, Mononen N, et al. 5-HTR1A, 5-HTR2A, 5-HTR6, TPH1 and TPH2 polymorphisms and major depression. Neuroreport 2009;20:1125-1128.

27. Farmer RF, Seeley JR. Temperament and character predictors of depressed mood over a 4-year interval. Depress Anxiety 2009;26:371-381.

28. Spittlehouse JK, Pearson JF, Luty SE, Mulder RT, Carter JD, McKenzie JM, et al. Measures of temperament and character are differentially impacted on by depression severity. J Affect Disord 2010;126:140-146.

29. Kampman O, Poutanen O, Illi A, Setala-Soikkeli E, Viikki M, Nuolivirta $\mathrm{T}$, et al. Temperament profiles, major depression, and response to treatment with SSRIs in psychiatric outpatients. Eur Psychiatry 2012; 27:245-249.

30. Kusunoki K, Sato T, Taga C, Yoshida T, Komori K, Narita T, et al. Low novelty-seeking differentiates obsessive-compulsive disorder from major depression. Acta Psychiatr Scand 2000;101:403-405. 